\title{
Epidermoid cysts of the cavernous sinus: clinical features, surgical outcomes, and literature review
}

\author{
*Feng Zhou, MD, PhD, ${ }^{1}$ Zixiao Yang, MD, PhD, ${ }^{1}$ Wei Zhu, MD, PhD, ${ }^{1}$ Liang Chen, MD, PhD, ${ }^{1}$ \\ Jianping Song, MD, PhD, ${ }^{1}$ Kai Quan, MD, PhD, ${ }^{1}$ Sichen Li, MD, ${ }^{1}$ Peiliang Li, MD, PhD, ${ }^{1}$ \\ Zhiguang Pan, MD, PhD, ${ }^{1}$ Peixi Liu, MD, PhD, ${ }^{1}$ and Ying Mao, MD, PhD ${ }^{1,2}$
}

\begin{abstract}
1Department of Neurosurgery, Huashan Hospital; and ${ }^{2}$ State Key Laboratory of Medical Neurobiology, School of Basic Medical Sciences and Institutes of Brain Science, Fudan University, Shanghai, People's Republic of China
\end{abstract}

\begin{abstract}
OBJECTIVE Epidermoid cysts of the cavernous sinus (CS) are rare, and no large case series of these lesions has been reported. In this study, the authors retrospectively reviewed the outcomes of the surgical management of CS epidermoid cysts undertaken at their center and performed a review of any such cysts reported in the literature over the past 40 years.

METHODS Clinical data were obtained on 31 patients with CS epidermoid cysts that had been surgically treated at the authors' hospital between 2001 and 2016. The patients' medical records, imaging data, and follow-up outcomes were retrospectively analyzed. The related literature from the past 40 years (18 articles, 20 patients) was also evaluated.

RESULTS The most common chief complaints were facial numbness or hypesthesia (64.5\%), absent corneal reflex $(45.2 \%)$, and abducens or oculomotor nerve deficit (35.5\%). On MRI, $51.6 \%$ of the epidermoid cysts showed low T1 signals and equal or high T2 signals. In the other lesions, the radiological findings varied considerably given differences in the composition of the cysts. Surgery was performed via the extradural approach (58.1\%), intradural approach (32.3\%), or a combined approach (9.7\%). After the operation, symptoms remained similar or improved in $90.3 \%$ of patients and new oculomotor paralysis developed after the operation in $9.7 \%$ of patients. Seven patients $(22.6 \%)$ developed meningitis postoperatively ( 5 aseptic and 2 septic), and all of them recovered. All patients achieved good recovery before discharge (Karnofsky Performance Status score $\geq 70$ ). Over an average follow-up of $4.6 \pm 3.0$ years in 25 patients $(80.6 \%)$, no recurrence or reoperation occurred, regardless of whether total or subtotal resection of the capsule had been achieved.
\end{abstract}

CONCLUSIONS Both the extradural and intradural approaches can enable satisfactory lesion resection. A favorable prognosis and symptomatic improvement can be expected after both total and subtotal capsule resections. Total capsule resection is encouraged to minimize the possibility of recurrence provided that the resection can be safely performed. https://thejns.org/doi/abs/10.3171/2017.6.JNS163254

KEY WORDS cavernous sinus; diffusion-weighted imaging; epidermoid cyst; middle cranial fossa; Meckel's cave; surgery; oncology

$\mathrm{E}$ PIDERMOID cysts are congenital, slow-growing lesions. On pathological examination, they appear as benign, pearly cystic lesions lined with simple stratified keratinizing squamous epithelium..$^{5,6,29}$ They are thought to originate from embryonic rests of ectodermal remnants that fail to undergo regression after the completion of neural embryogenesis in the fifth week of gestation. ${ }^{32}$ Some of these cells can remain entrapped in the meninges around nerves in the lateral wall of the cavern- ous sinus (CS) during embryonic development and subsequently give rise to epidermoid cysts. ${ }^{31}$ These cysts are uncommon, accounting for only $0.2 \%-1.8 \%$ of all intracranial tumors. ${ }^{5,21,31}$ Common locations for these lesions are the cerebellopontine angle (CPA), prepontine cistern, parasellar area, and middle cranial fossa (MCF). ${ }^{1}$ Epidermoid cysts originating from the CS are even rarer, ${ }^{7,17,24,26}$ hence, limited data are available on their surgical outcomes, and no large case series of such cysts has been reported. We,

ABBREVIATIONS CPA = cerebellopontine angle; CS = cavernous sinus; DWI = diffusion-weighted imaging; IF = infratemporal fossa; KPS = Karnofsky Performance Status; $M C F=$ middle cranial fossa; $P A=$ petrous apex; $P C F=$ posterior cranial fossa; $P F=$ pterygopalatine fossa.

SUBMITTED April 5, 2017. ACCEPTED June 19, 2017.

INCLUDE WHEN CITING Published online December 22, 2017; DOI: 10.3171/2017.6.JNS163254.

* Drs. Zhou and Yang contributed equally to this work. 
TABLE 1. Clinical characteristics of patients with epidermoid cysts of the cavernous sinus

\begin{tabular}{|c|c|}
\hline Characteristic & Value \\
\hline Mean age in yrs & $46.2 \pm 14.8$ \\
\hline \multicolumn{2}{|l|}{ Sex } \\
\hline Male & 16 \\
\hline Female & 15 \\
\hline \multicolumn{2}{|l|}{ Lesion side } \\
\hline Rt & 15 \\
\hline $\mathrm{Lt}$ & 16 \\
\hline \multicolumn{2}{|l|}{ Symptoms } \\
\hline Facial numbness or hypesthesia & $20(64.5 \%)$ \\
\hline Absent corneal reflex & $14(45.2 \%)$ \\
\hline Diplopia & $11(35.5 \%)$ \\
\hline Temporal muscle atrophy & $10(32.3 \%)$ \\
\hline Abducens nerve paralysis & $9(29.0 \%)$ \\
\hline Oculomotor nerve paralysis & $5(16.2 \%)$ \\
\hline TN & $3(9.7 \%)$ \\
\hline Facial paralysis & $2(6.5 \%)$ \\
\hline \multicolumn{2}{|l|}{ Location } \\
\hline \multicolumn{2}{|l|}{ w/o PCF invasion } \\
\hline MCF only & $13(41.9 \%)$ \\
\hline MCF \& IF & $2(6.5 \%)$ \\
\hline MCF \& PF & $2(6.5 \%)$ \\
\hline MCF, IF, \& PF & $1(3.2 \%)$ \\
\hline MCF \& suprasellar extension & $1(3.2 \%)$ \\
\hline MCF \& PA & $3(9.7 \%)$ \\
\hline \multicolumn{2}{|l|}{ w/ PCF invasion } \\
\hline MCF, PA, \& CPA & $7(22.6 \%)$ \\
\hline MCF, PA, IF, \& CPA & $2(6.5 \%)$ \\
\hline \multicolumn{2}{|l|}{ MRI features } \\
\hline T1, hypointense; T2, iso- or hyperintense lesion & $16(51.6 \%)$ \\
\hline T1, iso- or hyperintense; T2, hypointense lesion & $6(19.4 \%)$ \\
\hline T1 \& T2, mixed signal intensity & $9(29.0 \%)$ \\
\hline \multicolumn{2}{|l|}{ Surgical approach } \\
\hline Extradural only & $18(58.1 \%)$ \\
\hline Intradural only & $10(32.3 \%)$ \\
\hline Combined (intradural \& retrosigmoid) & $3(9.7 \%)$ \\
\hline \multicolumn{2}{|l|}{ Surgical results } \\
\hline Total capsule removal & $16(51.6 \%)$ \\
\hline Subtotal capsule removal & $13(41.9 \%)$ \\
\hline Subtotal capsule \& content removal & $2(6.5 \%)$ \\
\hline \multicolumn{2}{|l|}{ Postop meningitis } \\
\hline Aseptic & $5(16.1 \%)$ \\
\hline Septic & $2(6.5 \%)$ \\
\hline \multicolumn{2}{|l|}{ Surgical outcome before discharge } \\
\hline KPS score 70 & $1(3.2 \%)$ \\
\hline KPS score $\geq 80$ & $30(96.8 \%)$ \\
\hline FU & $25(80.6 \%)$ \\
\hline Mean duration in yrs & $4.6 \pm 3.0$ \\
\hline KPS score $\geq 80$ & $25(100 \%)$ \\
\hline Recurrence or reop & $0(0 \%)$ \\
\hline
\end{tabular}

CONTINUED IN NEXT COLUMN 》

\section{» CONTINUED FROM PREVIOUS COLUMN}

TABLE 1. Clinical characteristics of patients with epidermoid cysts of the cavernous sinus

$\mathrm{FU}=$ follow-up; $\mathrm{TN}=$ trigeminal neuralgia.

therefore, performed a retrospective study of our experience in treating such cysts. Additionally, we performed a literature review of all cases of CS epidermoid cysts reported over the past 40 years. Herein, we report the findings of both our case series and the literature review.

\section{Methods \\ Patients}

We screened the medical data of 726 patients with epidermoid cysts who had been treated in Huashan Hospital, Fudan University, between January 1, 2001, and June 30, 2016. Only patients with epidermoid cysts located in the lateral wall of the $\mathrm{CS}$, inside the $\mathrm{CS}$, and/or protruding toward the subtentorial space via Meckel's cave were included in our analysis. Thus, a total of 31 patients with 31 lesions were enrolled in this study, accounting for $4.3 \%$ of all epidermoid cyst cases at our center during the study period. All 31 cysts had been resected. The operations had been performed by 6 different senior professors at our center over the long study period. We performed a complete review of the medical data for all 31 patients, including their symptoms, original MRI findings, cyst location, pathological findings, surgical record, and follow-up data. At least 1 MRI study and an outpatient follow-up were recommended each year after surgery, but compliance was not ideal. Therefore, follow-up data were acquired from outpatient records or phone call interviews, and the judgment of radiographic lesion recurrence was based on the very last follow-up MRI study and imaging reports. Our study was approved by the ethics committee of Huashan Hospital, Fudan University. Given the retrospective nature of the study, informed consent was waived.

\section{Literature Review}

We also performed a literature search of PubMed for articles on CS epidermoid cysts that had been published in the last 40 years. Given the high probability of Meckel's cave invasion by epidermoid cysts in the CS, our search strategy was as follows: "epidermoid [title/abstract]" and "cavernous sinus [title/abstract]", "epidermoid [title/abstract]" and "Meckel's cave [title/abstract]", "cholesteatoma [title/abstract]" and "cavernous sinus [title/abstract]", "cholesteatoma [title/abstract]" and "Meckel's cave [title/ abstract]." There were 36 results based on this search strategy. After narrowing down the search results by reading the titles, abstracts, and/or full texts of the studies retrieved, we ended up with 18 articles.

\section{Results \\ Clinical Characteristics}

The clinical characteristics of the 31 patients in our case series are shown in Table 1 . There were 16 male and 
TABLE 2. Clinical outcomes with different surgical approaches

\begin{tabular}{|c|c|c|c|c|c|c|}
\hline \multirow[b]{2}{*}{ Surgical Approach } & \multicolumn{2}{|c|}{ PCF Invasion } & \multicolumn{3}{|c|}{ Surgical Results } & \multirow[b]{2}{*}{$\begin{array}{c}\text { New Postop } \\
\text { Neurological Deficit }\end{array}$} \\
\hline & No & Yes & $\begin{array}{l}\text { Total Capsule } \\
\text { Removal }\end{array}$ & $\begin{array}{l}\text { Subtotal Capsule } \\
\text { Removal }\end{array}$ & $\begin{array}{l}\text { Subtotal Capsule \& } \\
\text { Content Removal }\end{array}$ & \\
\hline Extradural only & 15 & 3 & 11 & 6 & 1 & 2 (recovered later on FU) \\
\hline Intradural only & 7 & 3 & 5 & 4 & 1 & 1 (lost to FU) \\
\hline Combined (intradural \& retrosigmoid) & 0 & 3 & 0 & 3 & 0 & 0 \\
\hline
\end{tabular}

15 female patients with a mean age of $46.2 \pm 14.8$ years (range 4-66 years). The lesion was located on the right in 15 patients and on the left in 16 . The majority of the patients' chief complaints were attributable to trigeminal nerve impairment, for example, facial numbness or hypesthesia (20 patients [64.5\%]), absent corneal reflex (14 patients [45.2\%]), temporal muscle atrophy (10 patients [32.3\%]), and trigeminal neuralgia (3 patients [9.7\%]). The second most common symptoms were those caused by abducens or oculomotor nerve deficit, such as diplopia (11 patients [35.5\%]), abducens paralysis (9 patients [29.0\%]), and oculomotor paralysis (5 patients [16.1\%]). Facial paralysis was seen in 2 patients $(6.5 \%)$ with subtentorial expansion of the lesion. None of the patients had hearing loss. Other symptoms included dizziness (5 patients [16.1\%]), mild limb weakness (1 patient [3.2\%]), and visual field defect (1 patient [3.2\%]).

\section{MRI Findings}

All patients had undergone MRI examination. The findings were varied but could be broadly divided into 3 categories according to the relative signal intensity of the lesion compared with that of the cerebral cortex on T1- and T2-weighted imaging: 1) low signal intensity on T1-weighted images and high or equal signal intensity on T2-weighted images (16 cases [51.6\%]; high intensity, 15 cases; equal intensity, 1 case); 2) equal or high signal intensity on T1-weighted images and low signal intensity on T2-weighted images (6 cases [19.4\%]; high intensity, 5 cases; equal intensity, 1 case); and 3) mixed signal intensity on both T1- and T2-weighted images (9 cases [29.0\%]). On contrast-enhanced imaging, all lesions showed heterogeneous enhancement of the cyst wall. Additionally, 13 patients underwent diffusion-weighted imaging (DWI). In 7 of these patients, the lesions showed high density as well as high T2 signals, and in the other 6 patients, the lesions showed low density with low $\mathrm{T} 2$ signals. One patient underwent digital subtraction angiography to differentiate the lesion from an aneurysm (case 2). In the illustrative cases below, we describe these 3 types of MRI presentations through 3 typical cases.

\section{Lesion Locations}

Given the lifelong and slow progression of epidermoid cysts, the lesions were usually large $(>3 \mathrm{~cm}, 23$ cases [74.2\%]). Twenty-two lesions $(71.0 \%)$ were located entirely supratentorially without posterior cranial fossa (PCF) invasion, whereas the other 9 lesions (29.0\%) were located supratentorially but also exhibited PCF invasion
(Table 1). The major component of the lesion was located in the parasellar region in the MCF. Among the 22 lesions that showed no subtentorial expansion, 13 were located in the MCF only, 5 showed pterygopalatine fossa (PF) and/ or infratemporal fossa (IF) protrusion, 1 showed suprasellar extension (case 1), and 3 exhibited petrous apex (PA) extension via Meckel's cave. The remaining 9 lesions extended subtentorially to the CPA via the dilated Meckel's cave and eroded the PA corridor; 2 of these lesions also showed IF extension.

\section{Surgical Strategies and Outcomes}

The surgical approaches used in our series were divided into 3 types: extradural, intradural, and combined extra- and intradural (Table 2). An extradural approach to the MCF was used in 18 patients $(58.1 \%)$, including 15 without subtentorial protrusion and 3 with PCF invasion. The extradural approach was achieved via frontotemporal orbitozygomatic (5 cases), frontotemporal zygomatic (12), or subtemporal (1) craniotomy. Lumbar puncture drainage was usually performed to lower intracranial pressure during the operation and was discontinued after the operation. The temporal lobe was lifted via extradural retraction to expose the lateral wall of the CS. This wall was incised to expose and resect the cyst contents and capsule. In some patients, even lesions that extended to the subtentorial space could be resected through the dilated Meckel's cave via this approach. More recently, a high-resolution endoscope (Karl Storz Endoskope) has been used at our center for more radical resections. This instrument was used in 3 patients in the current series (2 lesions located supratentorially and 1 with subtentorial protrusion), and in 1 of the patients without PCF invasion, the lesion was removed entirely endoscopically via the infratemporal extradural approach. After lesion removal, the lateral wall of the CS was tightly sutured and sealed with fibrin glue. Total capsule removal was achieved in 11 patients (including 3 cases with endoscope assistance), subtotal capsule removal was achieved in 6 patients, and subtotal capsule and content removal was achieved in 1 patient.

The intradural approach was used in 10 patients $(32.3 \%)$, including 7 without subtentorial protrusion and 3 with minimal subtentorial invasion. This approach was selected for patients with large lesions, most of which were located supratentorially and showed upward and lateral extension. After frontotemporal craniotomy, the bulging but intact meningeal dura of the CS could be exposed through careful Sylvian fissure and temporal pole dissection. In this way, the CS could be entered from its upper 
wall, and lesion removal could be performed. The CS wall was tightly sutured after lesion removal and sealed with fibrin glue before dura closure. Total capsule removal, subtotal capsule removal, and subtotal capsule and content removal were achieved in 5, 4, and 1 patient, respectively. However, although it was difficult to make a statistical analysis, compared with surgical results via the extradual approach (2 total capsule resection and 1 subtotal capsule resection), only a less radical resection could be made via the intradural approach (2 subtotal capsule resections, 1 subtotal capsule and content removal) for those cases with PCF occupation.

The combined supratentorial intradural and suboccipital (retrosigmoid) approach was used in 3 patients with large MCF and PCF masses. Despite the more extensive surgical exposure via this approach, only subtotal capsule removal could be achieved in all 3 patients because of the widespread, tight adhesions to nearby neurological and vascular structures, especially the brainstem.

There was no standardized protocol for the use of perioperative steroids. Seventeen patients were treated with dexamethasone (5-15 $\mathrm{mg}$ /day intravenously) postoperatively for 1-10 days, while the other 14 patients did not accept any perioperative steroid treatment. There were no fatal surgical complications in this series. Meningitis developed in 7 patients postoperatively, 2 of whom had septic meningitis; the other 5 patients had aseptic meningitis. There was no significant statistical relationship between aseptic meningitis and postoperative steroid treatment ( $p$ $=1.000)$ or the extent of capsule removal $(\mathrm{p}=0.172)$. In all 7 patients, the meningitis was resolved before discharge.

All 31 patients achieved good recovery before dis- charge (Karnofsky Performance Status [KPS] score $\geq 70$ ). In 28 patients $(90.3 \%)$, the symptoms remained similar (23) or improved (5) after surgery, but in 3 patients (9.7\%), new oculomotor paralysis developed after the operation. Twenty-five patients (80.6\%) attended follow-up assessments for a mean of $4.6 \pm 3.0$ years (range $0.1-10.2$ years) and had KPS scores $\geq 80$. None of them reported the radiographic recurrence of an epidermoid cyst or required reoperation. Nearly half of them (11 patients) showed further improvement after discharge, which mostly consisted of obvious remission of diplopia or oculomotor paralysis (9 patients). Of the 3 patients with new oculomotor paralysis after the operation, 1 was lost to follow-up. The other 2 patients eventually attained complete spontaneous recovery (Table 2 ).

\section{Illustrative Cases \\ Case 1}

A 36-year-old woman presented with a history of decreasing vision over 20 years and dizziness for 2 months. A neurological examination revealed strabismus in the left eye. Magnetic resonance imaging revealed a left CS lesion with suprasellar extension. The lesion was hypointense on T1-weighted imaging and hyperintense on T2-weighted imaging (Fig. 1A and B). A slightly enhanced rim was found at the periphery of the lesion on contrast-enhanced MRI (Fig. 1C-E). On DWI, the lesion was hyperintense, which helped to distinguish it from an arachnoid cyst (Fig. $1 \mathrm{~F})$. The patient underwent a left frontotemporal zygomatic craniotomy, and the lateral wall of the CS was exposed extradurally. The lesion was located between the V1 and
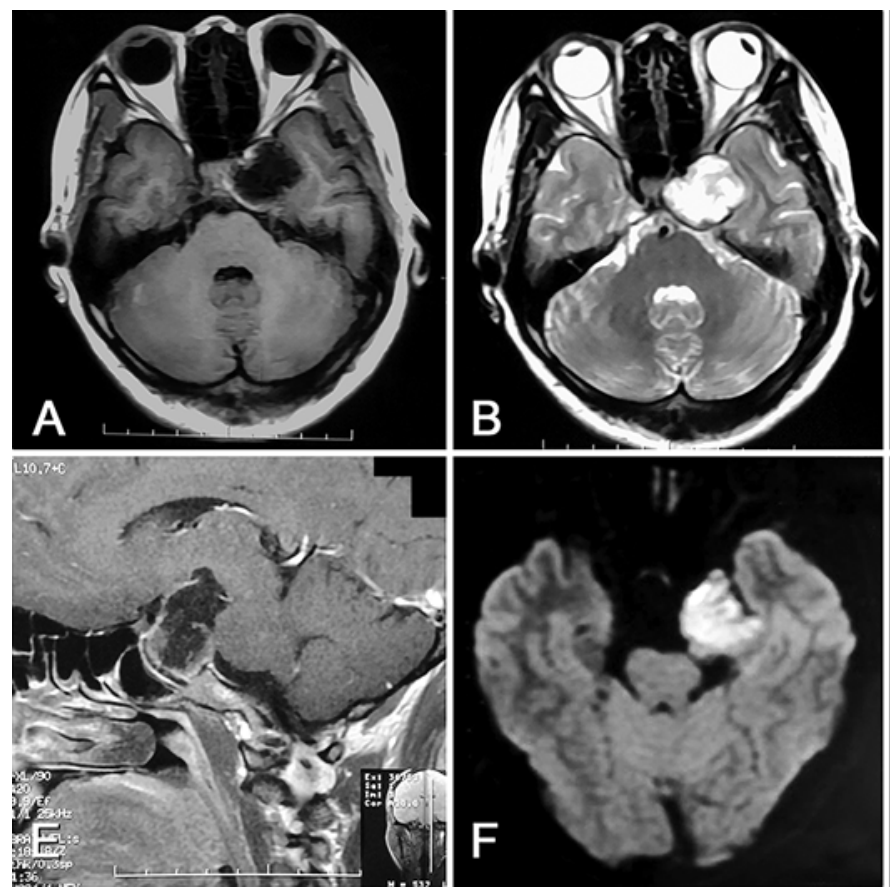
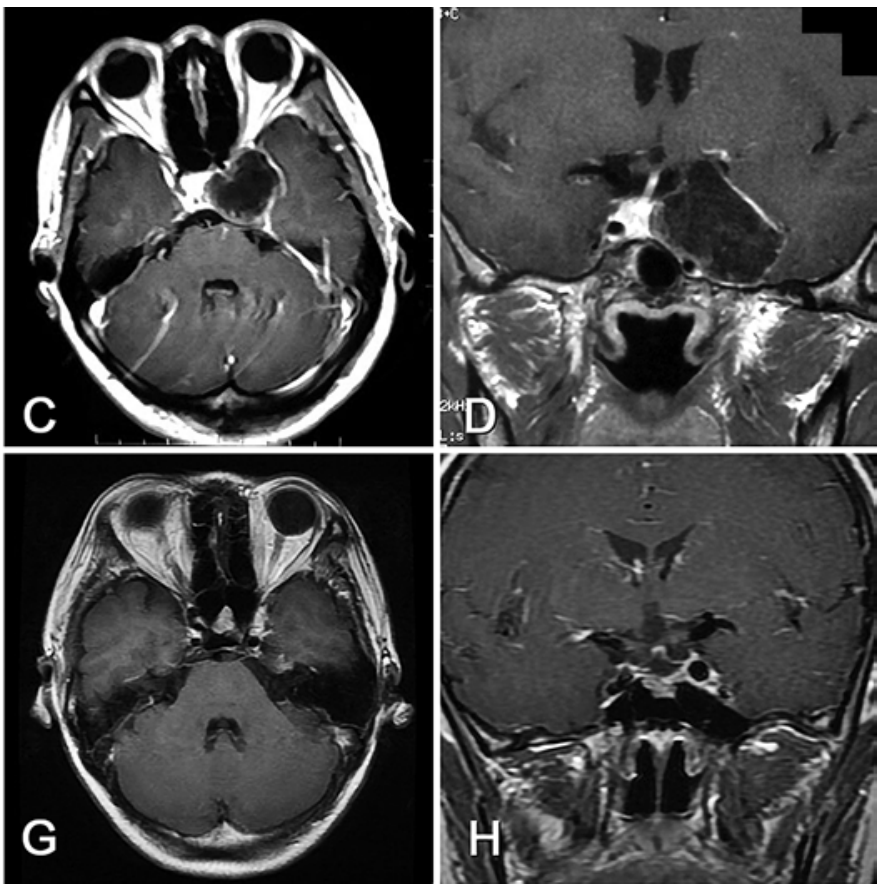

FIG. 1. Case 1. The lesion appeared hypointense on T1-weighted imaging (A) and hyperintense on T2-weighted imaging (B). Hyperintense signals appeared around the lesion on enhanced T1-weighted imaging $(\mathbf{C}-\mathbf{E})$. The lesion appeared hyperintense on DWI $(\mathbf{F})$, which helped to distinguish it from an arachnoid cyst. No recurrence was observed on the follow-up MR images (G and $H)$. 
$\mathrm{V} 2$ branches of the trigeminal nerve. Total removal of the cyst contents and its capsule was achieved. No new neurological deficits occurred after the operation. A 3-year follow-up revealed no recurrence of symptoms or the imaging findings (Fig. $1 \mathrm{G}$ and $\mathrm{H}$ ).

\section{Case 2}

A 63-year-old man reported a 3-year history of intermittent headaches and a 6-month history of left facial numbness. He began experiencing severe left facial stabbing pain 1 week before admission to our hospital. A neurological examination revealed slight left blepharoptosis, negative left corneal reflex, and decreased pinprick sensation on the left side of his face. Computed tomography showed a high-density lesion in the left CS (Fig. 2A). On MRI, the lesion appeared predominantly hyperintense on T1-weighted imaging but hypointense on T2-weighted imaging (Fig. 2B and C). The lesion appeared hypointense on DWI (Fig. 2D). Therefore, a giant thrombosed CS aneurysm was highly suspected. Computed tomography angiography and digital subtraction angiography were then performed and proved that no aneurysm existed (Fig. 2E and F). The lesion was explored via a left frontotemporal zygomatic extradural approach and was seen to be located in the interdural space of the lateral wall of the CS. After total removal of the internal contents, the whitish capsule of the lesion was carefully dissected from the nearby neurovascular structures. The oculomotor, trochlear, and trigeminal nerves were well preserved during the operation, and complete capsule resection was achieved. Patho- logical examination of the resected specimen revealed that the lesion was an epidermoid cyst. The patient recovered with no complications. No recurrence was found during an 8-year follow-up.

\section{Case 3}

A 30-year-old man presented with dizziness and right temporal muscle atrophy, both of which he had experienced for several years. A neurological examination revealed that the corneal reflex was absent on the right side. Magnetic resonance imaging revealed a dumbbell-shaped lesion in the MCF and PCF. The lesion showed mixed hyper- and hypointense signals on both T1- and T2-weighted images (Fig. 3A and B). The cyst wall was heterogeneously enhanced (Fig. 3C and D). The lesion was approached and exposed extradurally via a right orbitozygomatic craniotomy (Fig. 3E). After removal of the lesion between the V1-2 and V2-3 spaces, the lesion in the PCF was removed via the expanded Meckel's cave. An endoscope was also used during the operation for radical lesion resection in the PCF. Because attachment of the tumor capsule to the brainstem was not prominent, total capsule resection could be achieved (Fig. 3F). After surgery, the patient developed an irregular fever (range $37.0^{\circ} \mathrm{C}-39.5^{\circ} \mathrm{C}$ ), which lasted for 2 months. Cerebrospinal fluid examination revealed that the leukocyte count did not exceed $2500 \times 10^{6}$ cells $/ \mathrm{ml}$, which was consistent with the tendency of the fever to vary. The glucose level in the cerebrospinal fluid was normal at every examination (total of 12 examinations). Furthermore, no evidence of septic infection in the cerebrospinal fluid was
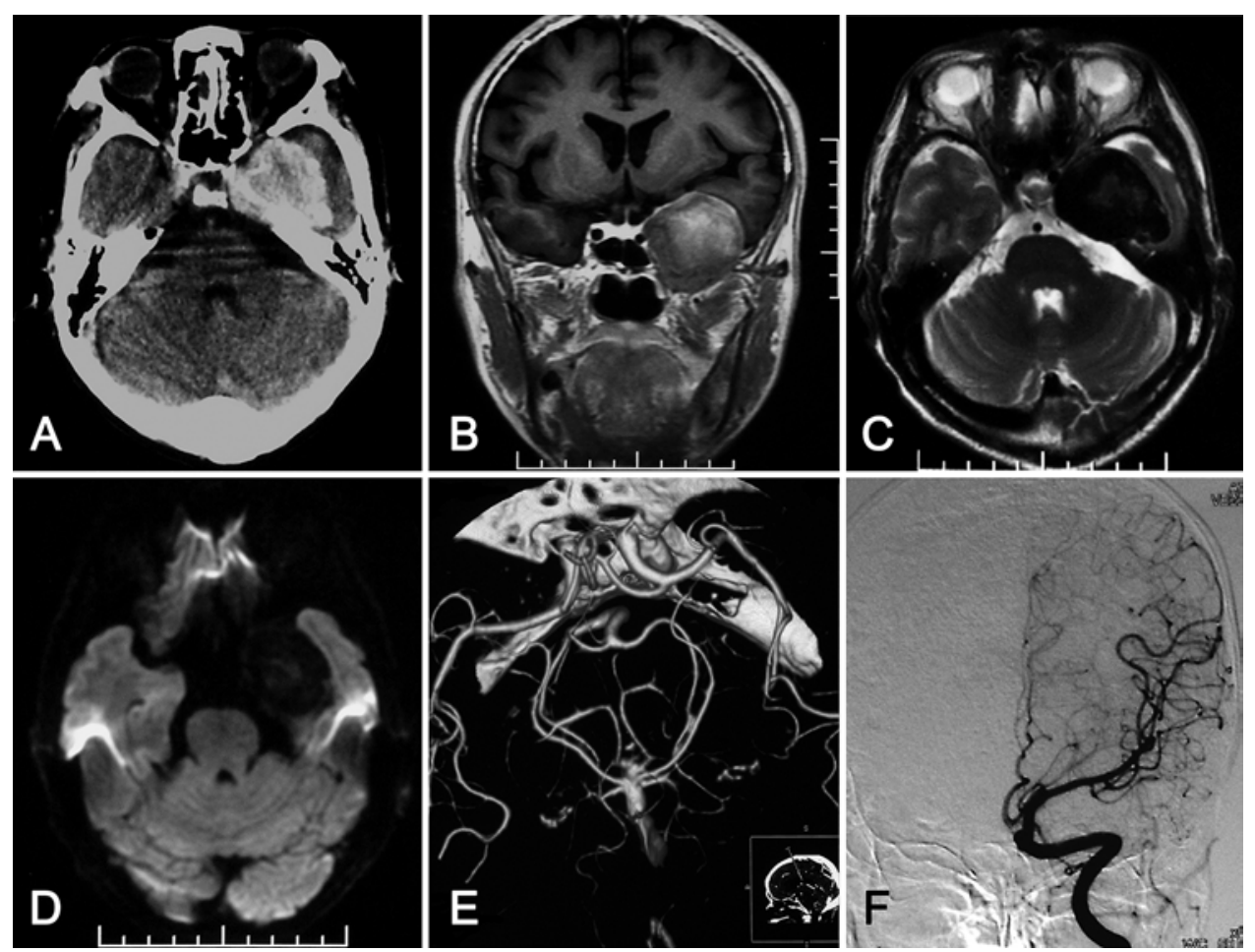

FIG. 2. Case 2. The lesion appeared hyperintense on $C T(\mathbf{A})$ and T1-weighted imaging $(\mathbf{B})$, and a flow void sign (C) was seen on T2-weighted imaging. A hypointense lesion was observed on DWI (D). Computed tomography angiography (E) and digital subtraction angiography $(\mathbf{F})$ demonstrate no cerebral aneurysm and no staining of the lesion. 

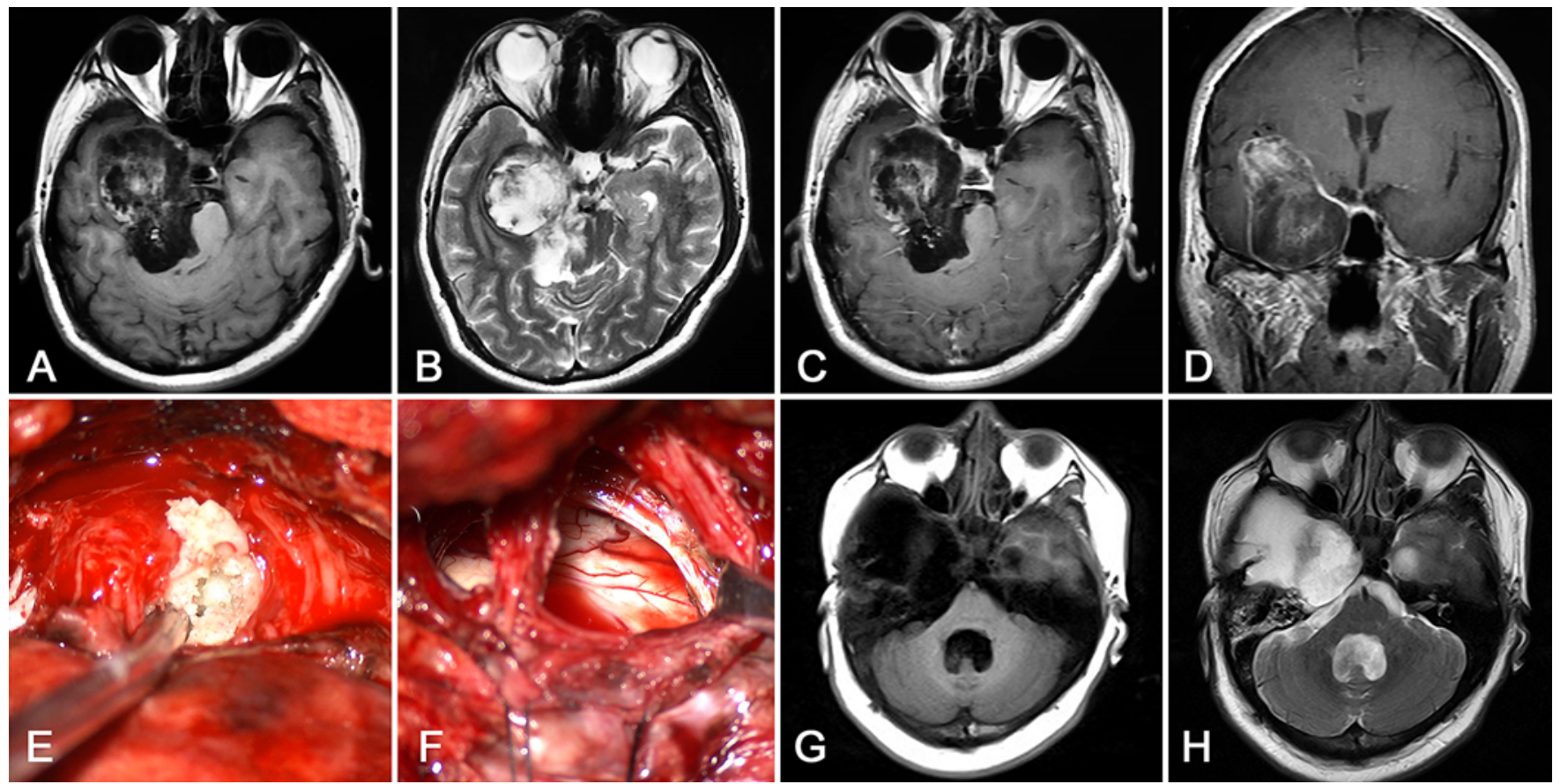

FIG. 3. Case 3. The lesion showed mixed signals on T1-weighted (A) and T2-weighted (B) images. Heterogeneous enhancement appeared on enhanced T1-weighted images ( $\mathbf{C}$ and $\mathbf{D})$. The lesion presented a typical cholesteatoma-like appearance $(\mathbf{E})$ and was completely removed $(\mathbf{F})$. No recurrence was observed on follow-up MR images ( $\mathbf{G}$ and $\mathbf{H})$. Figure is available in color online only.

found. The patient recovered with supportive treatment. A 4-year follow-up showed no recurrence (Fig. 3G and H).

\section{Literature Review}

We reviewed all the literature on CS epidermoid cysts from the past 40 years. In total, 18 reports including 20 patients were retrieved and analyzed (Table 3). The patients consisted of 8 males $(40 \%)$ with a mean age of $44.63 \pm$ 22.42 years and 12 females with a mean age of $32.58 \pm 9.14$ years. Most of the chief complaints were attributable to trigeminal nerve impairment, for example, facial numbness or hypesthesia (12 patients [60\%]), trigeminal neuralgia (5 patients [25\%]), absent corneal reflex (2 patients [10\%]), and temporal muscle atrophy (1 patient [5\%]). The second most common types of complaint were those caused by abducens or oculomotor nerve deficits, including diplopia (7 patients [35\%]), abducens paralysis (7 patients [35\%]), and oculomotor paralysis (8 patients [40\%]). Additionally, 8 patients (40\%) complained of headache. All patients underwent preoperative CT or MRI, which revealed that 9 lesions $(45 \%)$ were on the left side. Surgery was performed via the extradural approach in 6 patients $(30 \%)$ and the intradural approach in 9 patients (45\%), and 1 patient (5\%) underwent surgery twice, first via the intradural approach and via the extradural-intradural approach in the second operation. Four cases $(20 \%)$ lacked detailed information regarding an extradural or intradural approach. Total capsule resection was achieved in 4 patients $(20 \%)$, and subtotal resection was achieved in $10(50 \%)$; there was no information about the extent of surgery in the other 6 patients $(30 \%)$. Generally, the outcome of the patients was satisfactory. During a follow-up of $2.08 \pm 1.77$ years, only 2 patients (10\%) showed recurrence.

\section{Discussion}

Intracranial epidermoid cysts are an uncommon finding, $, 521,31$ and epidermoid cysts of the CS are even rarer, accounting for only $2 \%$ of all intracranial epidermoid tumors. ${ }^{31}$ At our center, lesions of the CS account for $4.3 \%$ of all epidermoid cysts. Few cases of CS epidermoid cysts have been reported in the literature, and no large case series has been published as yet. ${ }^{1,3,5,7-11,14-17,20-22,24,29,31}$ In the current study, we have presented the first and largest series of epidermoid cysts in the CS.

\section{Clinical Characteristics}

Most patients in our series as well as those in the studies included in the literature review presented with symptoms of facial numbness or hypesthesia, absent corneal reflex, diplopia, temporal muscle atrophy, ptosis, limited eye movement, trigeminal neuralgia, headache, and so forth (Tables 1 and 3). These symptoms are caused by compression of the surrounding nervous structures by the epidermoid cyst, especially the trigeminal, oculomotor, and trochlear nerves. Consistent with findings in the literature, the main cause of symptoms in our patients was trigeminal nerve impairment, followed by abducens or oculomotor nerve deficit.

\section{Radiological Features}

Head CT and MRI are the primary diagnostic meth- 


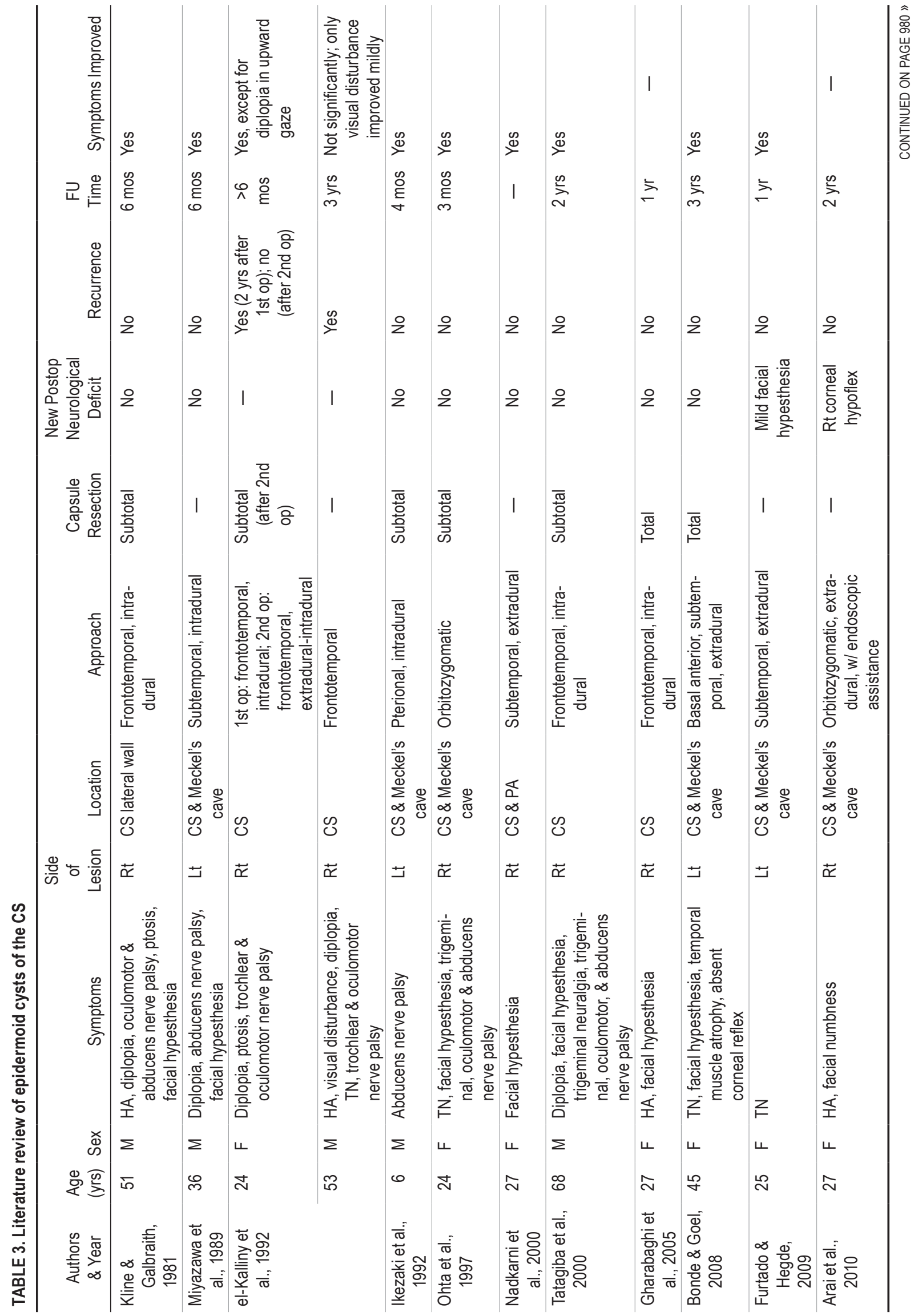




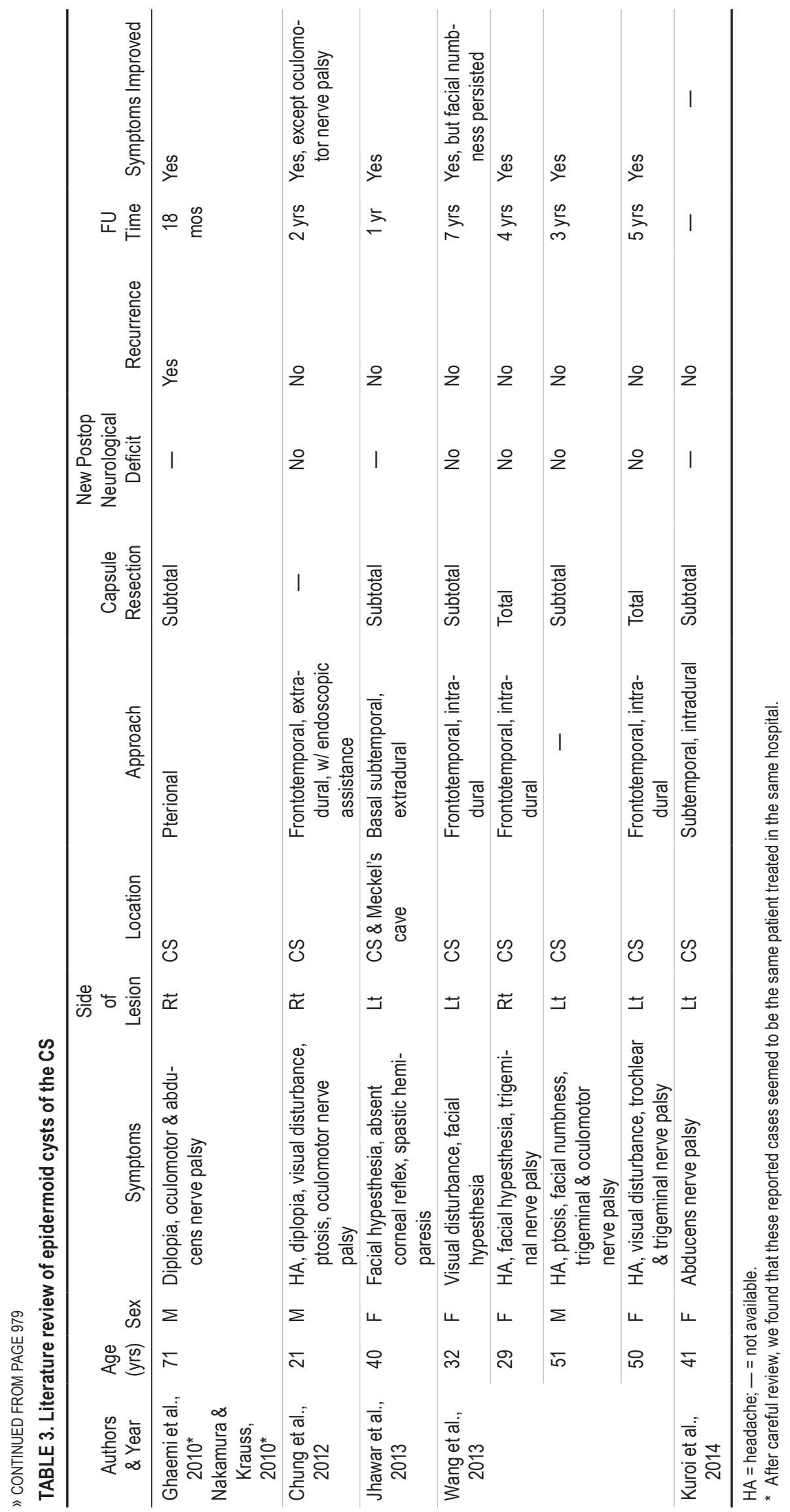


ods for epidermoid cysts in the CS. Computed tomography scans usually showed a low-density space-occupying mass with slight peripheral enhancement. ${ }^{16,20,29}$ However, diverse findings were obtained on MRI examinations given the variation in the composition of the epidermoid cysts. ${ }^{1}$ The MRI findings could be broadly divided into 3 categories: 1) low signal intensity on T1-weighted images and high or equal signal intensity on T2-weighted images; 2) equal or high signal intensity on T1-weighted images and low signal intensity on T2-weighted images; and 3) mixed signal intensity on both T1- and T2-weighted images. The first category of findings was usually caused by cysts that mainly contained white, waxy material rich in cholesterol crystals. ${ }^{1}$ These lesions were the most common type of epidermoid cyst in our series (16 cases [51.6\%]). Such cysts appear hyperintense on DWI and T2-weighted images, which differentiates them from arachnoid cysts (case 1). ${ }^{12}$

The second category of findings-hyperintensity on T1-weighted images and hypointensity on T2-weighted images - may be attributable to the high protein concentrations and viscosity of the cysts. Such cysts may appear hyperdense on $\mathrm{CT}^{1,2,31}$ and hypointense on T2-weighted MRI and DWI.,18 In our series, 6 patients (19.4\%) showed these radiological features. As illustrated in case 2, because of the location of the CS, this type of epidermoid cyst can be misdiagnosed as a giant internal carotid artery aneurysm. Further examinations, such as cerebral angiography, should be performed to clarify the diagnosis in these cases.

The third category of MRI findings consisted of mixed hyperintense and hypointense signals on T1- and T2weighted images. ${ }^{4,13,23}$ In addition to the original contents of the epidermoid cysts, hemorrhage or granuloma tissue can also occur inside the cysts with lesion progression. ${ }^{4,13,25,30}$ In our series, 9 patients (29.0\%) exhibited such a mixed radiological appearance. These findings must be differentiated from lesions such as neurinoma or cholesterol granuloma. ${ }^{9,27}$ Occasionally, however, it is difficult to make an accurate preoperative diagnosis based on the MRI data, especially if the epidermoid cyst extends into the PCF via Meckel's cave, as this radiological appearance very closely mimics a trigeminal neurinoma (case 3 ).

\section{Classification of Epidermoid Cysts of the CS}

In 1992, el-Kalliny et al. classified CS tumors into 3 types: intracavernous, invasive, and interdural. ${ }^{8}$ In 2005 , Gharabaghi et al. divided epidermoid tumors of the CS into 3 groups: extracavernous, interdural, and intracavernous. ${ }^{11}$ The extracavernous group consists of epidermoid cysts that originate from extracavernous tissue and invade or compress the CS. In this group, the cyst is frequently located in Meckel's cave and arises from the PA. The interdural group consists of tumors that are located between the 2 dural layers of the lateral wall of the CS. "True" intracavernous group tumors are located between the inner membranous layers of the CS and tend to encase the internal carotid artery, encircling and displacing the cranial nerves laterally. ${ }^{11}$

We tried to divide our series into the 3 groups described by Gharabaghi et al. However, we found that there were too many large lesions ( $>3 \mathrm{~cm}, 23$ cases [74.2\%]), so it was dif- ficult to clearly distinguish the relationship between the lesion and the different dural layers on MRI. Moreover, about one-third of the lesions showed subtentorial invasion via the dilated Meckel's cave. In these cases, it was difficult to determine if the lesions were of true extracavernous origin or if they grew from the interdural space into the CPA along the trigeminal nerve. Therefore, we categorized the lesions according to whether they exhibited PCF invasion, which determined our choice of surgical approach (Table 1).

In our series, 22 lesions (71.0\%) were purely supratentorial, and 9 (29.0\%) exhibited PCF invasion. Of the 22 supratentorial lesions, 13 were confined to the MCF, while 5 showed IF or PF protrusion. One lesion (3.2\%) showed suprasellar extension and was associated with visual deficit. We found that 12 lesions (38.7\%) had invaded Meckel's cave, occupying the PA area without PCF invasion (3 lesions) or even extending into the CPA (9 lesions). Among the 20 cases included in the literature review, $7(35 \%)$ had a clear description about the location of the lesion relative to Meckel's cave, and in 1 case, PA invasion was mentioned (Table 3).

\section{Surgical Approaches}

Because of their congenital and benign nature, epidermoid cysts do not require surgery if they are asymptomatic. Yanamadala et al. even described a case of spontaneous regression of an epidermoid cyst of the CS. ${ }^{32}$ However, most symptomatic epidermoid cysts are treated with surgery, which is an efficient treatment strategy for these tumors. ${ }^{11}$ The surgical approaches used can be either intraor extradural. ${ }^{22}$ Frontotemporal or pterional craniotomy and their modifications, such as the inclusion of the zygomatic arch with or without partial orbital plate removal or only subtemporal entry, were commonly used in the reports included in the literature review (Table 3 ) and in our study (Table 1).

On the basis of our experience, we consider the extradural approach to be beneficial for the resection of CS tumors. ${ }^{19,33,34}$ Therefore, unlike most cases $(52.4 \%)$ in the reviewed literature, which involved intradural tumor resection (Table 3), tumor removal was achieved via the extradural approach in most of our patients (58.1\%). Determining whether to perform the orbitozygomatic or zygomatic extradural approach depends on the degree of lesion extension into the PCF. If there was more lateral extension in the PCF, the frontotemporal orbitozygomatic approach was preferred for wider visualization. The lateral wall of the CS can be well exposed after lifting the temporal lobe extradurally, and the interdural space can be easily exposed by mobilizing the first and second branches of the trigeminal nerve. Tumors located interdurally can be efficiently excised between these two branches. Even true intracavernous lesions can be further exposed by opening the inner layer of the CS. By dissecting the dura over the third branch of the trigeminal nerve and ganglion, Meckel's cave can be entered via the porus trigeminus. Subtentorial lesions can be exposed and resected via the dilated Meckel's cave. ${ }^{33,34}$ In our study, the extradural approach was used for 15 lesions without subtentorial protrusion and 3 lesions with PCF invasion, and total or subtotal capsule resection was achieved in 17 (94.4\%) of these lesions (Table 2). 
The intradural approach was the second most commonly used approach in patients with largely upward and lateral extension of the lesion (10 lesions). Total or subtotal capsule resection was achieved in $90 \%$ of such lesions in our series (Table 2). Although it was an effective method of lesion removal, our results also suggest that the extradural approach may be more suitable than the intradural approach for lesions occupying the PCF.

The combined approach was seldom used in our series. It was required in some patients with dumbbell-shaped lesions that were large in both the MCF and PCF and probably without enough of a surgical corridor through Meckel's cave. Only subtotal capsular removal could be achieved in such lesions (3 lesions [100\%]; Table 2) because of the tight adhesion of the tumor capsule to the brainstem. However, with the development of endoscope technology, we believe that endoscope-assisted resection may help to achieve similar surgical results using a single extradural approach rather than the combined approach in the future.

Actually, in our recent practice, radical tumor resection with total capsule removal was achieved using endoscopeassisted surgery via the extradural approach in 3 patients, including one in whom the tumor was resected entirely under endoscopy. A recent case report also showed the benefits of endoscope-assisted surgery for minimizing the risk of neurovascular injury and achieving better exposure for an epidermoid cyst of the CS. ${ }^{5}$ Although endoscopic resection is challenging and more experience is required, we believe that because of the clearer and wider exposure of the cyst contents and capsule, relatively less intraoperative bleeding, and the handy removal of the waxy contents of such lesions by simple suction, epidermoid cysts of the CS may be ideal for minimally invasive endoscopic surgery in the future.

\section{Extent of Resection}

The need for total capsule resection in patients with epidermoid cysts of the CS has been the subject of debate. Compared with authors of previous studies, we may have a more aggressive attitude toward tumor capsule resection. Total capsule resection was achieved in only $4(20.0 \%)$ of the 20 reported cases (Table 3) but in $16(51.6 \%)$ of the 31 patients in our series (Table 2), most of whom (14 patients [87.5\%]) did not have PCF invasion. Additionally, $2(12.5 \%)$ of the 16 patients with total capsule resection developed new neurological deficits after surgery, as compared with 1 patient (6.7\%) with subtotal capsule resection with or without subtotal content removal. This may indicate that more aggressive capsular resection leads to injuries to the cranial nerves in the CS. ${ }^{11}$ However, we found that the new postoperative neurological deficits resolved in both patients with total capsule resection. Among the previously reported cases, 2 patients developed new neurological deficits after surgery; however, the extent of capsule resection was not reported in those cases. Interestingly, 4 of the previously reported cases with total capsule resection as well as other patients with subtotal capsule resection had no new symptoms after surgery (Table 3). Most importantly, all available cases with follow-up data, both the previously reported cases and those in our series, had very satisfactory recoveries after surgery with continuing improvement after discharge (Tables 1 and 3). Therefore, considering the satisfactory postoperative outcomes and further improvement both in the literature and in our series, we suggest that both total and subtotal capsule resections are acceptable in patients with an epidermoid cyst of the CS. Given our experience, we recommend attempting total capsule resection for lesions that are primarily confined to the MCF without extensive PCF invasion because it may be very difficult and dangerous to aggressively resect the tumor capsule off complex neurovascular structures, especially the brainstem, in the PCF. ${ }^{28}$

There were no recurrences or reoperations in our series, but 3 recurrences $(1$ case did not recur after the second operation) were reported in the literature, including 2 in which subtotal capsule resection had been performed (Table 3). Thus, given the tendency for recurrence after subtotal resection, we encourage skilled surgeons to attempt total capsule resection in appropriate cases?

\section{Study Limitations}

There are some limitations to our study. First, the identification of total or subtotal capsule resection may not have been precise in each case because of the retrospective nature of our study. We think that only thick residual capsular tissue can be visualized under a microscope or an endoscope. If the capsule is too thin and transparent or tightly attached to nerves or vessels, we may not be able to identify it every time. Therefore, we believe that the rate of total capsule resection may have been overestimated in our series as well as in the previously reported cases. Second, because of the limited number of cases and the loss to follow-up, we were unable to obtain any useful statistical data. Neither could we perform statistical analysis of our cases and the previously reported cases together because of missing information in the latter cases. However, we do hope that the data we shared in this paper will provide useful information or inspire some new insights about this disease.

\section{Conclusions}

In this article, we have reported the largest case series on the surgical management of epidermoid cysts of the CS and have carefully reviewed the literature on this topic. The variation in radiological features of the epidermoid cysts was related to differences in the composition of the cystic contents and may make preliminary diagnosis difficult. Both extradural and intradural approaches could be used to achieve satisfactory lesion resection. Some new neurological deficits may occur after the operation, but their incidence was minimal in our study and most of them resolved during follow-up. Meningitis was the main postoperative complication but could be cured. Regardless of whether capsule resection is total or subtotal, satisfactory outcomes and continuous improvement in neurological symptoms can be expected. Total capsule resection can be attempted to minimize the possibility of recurrence. However, we recommend a safe surgical strategy over an aggressive one. The exact technique should be based on the surgeon's skills and proficiency in capsule resection 


\section{Acknowledgments}

This work was supported by the National Natural Science Foundation of China (No. 81400962 to J.S., No. 81571102 to W.Z.). Additional support covering most of the bibliographic retrieval services was contributed by the Science and Technology Commission of Shanghai Municipality (No. 16495800300 to Yanlong Tian).

\section{References}

1. Arai A, Sasayama T, Koyama J, Fujita A, Hosoda K, Kohmura E: Epidermoid cyst in Meckel's cave with unusual computed tomography and magnetic resonance imaging findings. Case report. Neurol Med Chir (Tokyo) 50:701704, 2010

2. Ben Hamouda M, Drissi C, Sebai R, Hammami N, Ghorbel D, Zammel I, et al: Atypical CT and MRI aspects of an epidermoid cyst. J Neuroradiol 34:129-132, 2007

3. Bonde $\mathrm{V}$, Goel A: Interdural cavernous sinus epidermoid cyst. J Clin Neurosci 15:212-214, 2008

4. Chen CY, Wong JS, Hsieh SC, Chu JS, Chan WP: Intracranial epidermoid cyst with hemorrhage: MR imaging findings. AJNR Am J Neuroradiol 27:427-429, 2006

5. Chung JC, Kim SM, Sade B, Kim HK, Park MS, Chung SY, et al: Endoscope-assisted microsurgical removal of an epidermoid tumor within the cavernous sinus. Yonsei Med J 53:1216-1219, 2012

6. Cobbs CS, Pitts LH, Wilson CB: Epidermoid and dermoid cysts of the posterior fossa. Clin Neurosurg 44:511-528, 1997

7. Dufour H, Fuentes S, Metellus P, Grisoli F: [Intracavernous epidermoid cyst. Case report and review of the literature.] Neurochirurgie 47:55-59, 2001 (Fr)

8. el-Kalliny M, van Loveren H, Keller JT, Tew JM Jr: Tumors of the lateral wall of the cavernous sinus. J Neurosurg 77:508-514, 1992

9. Furtado SV, Hegde AS: Trigeminal neuralgia due to a small Meckel's cave epidermoid tumor: surgery using an extradural corridor. Skull Base 19:353-357, 2009

10. Ghaemi K, Mirzayan MJ, Krauss JK: Interdural epidermoid of the cavernous sinus. Cent Eur Neurosurg 71:155-156, 2010

11. Gharabaghi A, Koerbel A, Samii A, Safavi-Abbasi S, Tatagiba M, Samii M: Epidermoid cysts of the cavernous sinus. Surg Neurol 64:428-433, 2005

12. Hakyemez B, Aksoy U, Yildiz H, Ergin N: Intracranial epidermoid cysts: diffusion-weighted, FLAIR and conventional MR findings. Eur J Radiol 54:214-220, 2005

13. Hsieh CH, Huang KM, Kao MC, Peng S, Wang CC: Hemorrhage in intracranial epidermoid cyst. J Formos Med Assoc 95:173-175, 1996

14. Ikezaki K, Toda K, Abe M, Tabuchi K: Intracavernous epidermoid tumor presenting with abducens nerve paresis-case report. Neurol Med Chir (Tokyo) 32:360-364, 1992

15. Jhawar SS, Mahore A, Goel AH: Giant multicompartmental epidermoid cyst of cavernous sinus: a case report. J Neurol Surg A Cent Eur Neurosurg 74 (Suppl 1):e9-e12, 2013

16. Kline LB, Galbraith JG: Parasellar epidermoid tumor presenting as painful ophthalmoplegia. J Neurosurg 54:113117,1981

17. Kuroi Y, Yoshimura C, Yokosako S, Arai N, Ohbuchi H, Hirota K, et al: [A case of cavernous sinus epidermoid: anatomical classification and surgical approach.] No Shinkei Geka 42:149-155, 2014 (Jpn)

18. Li F, Zhu S, Liu Y, Chen G, Chi L, Qu F: Hyperdense intracranial epidermoid cysts: a study of 15 cases. Acta Neurochir (Wien) 149:31-39, 2007

19. Liu XD, Xu QW, Che XM, Yang DL: Trigeminal neurinomas: clinical features and surgical experience in 84 patients. Neurosurg Rev 32:435-444, 2009

20. Miyazawa N, Yamazaki H, Wakao T, Nukui H: Epidermoid tumors of Meckel's cave: case report and review of the literature. Neurosurgery 25:951-955, 1989

21. Nadkarni T, Dindorkar K, Muzumdar D, Goel A: Epidermoid tumor within Meckel's cave-case report. Neurol Med Chir (Tokyo) 40:74-76, 2000

22. Nakamura M, Krauss JK: Image-guided resection of small lesions in the cavernous sinus and Meckel's cave. Eur J Surg Oncol 36:208-213, 2010

23. Ochi M, Hayashi K, Hayashi T, Morikawa M, Ogino A, Hashmi R, et al: Unusual CT and MR appearance of an epidermoid tumor of the cerebellopontine angle. AJNR Am J Neuroradiol 19:1113-1115, 1998

24. Ohta H, Ottomo M, Nakamura T, Yokota A: [A case of epidermoid tumor inside the Meckel's cave.] No Shinkei Geka 25:943-947, 1997 (Jpn)

25. Osborn AG, Preece MT: Intracranial cysts: radiologicpathologic correlation and imaging approach. Radiology 239:650-664, 2006

26. Pamir MN, Kilic T, Ozek MM, Ozduman K, Türe U: Nonmeningeal tumours of the cavernous sinus: a surgical analysis. J Clin Neurosci 13:626-635, 2006

27. Razek AA, Huang BY: Lesions of the petrous apex: classification and findings at $\mathrm{CT}$ and MR imaging. Radiographics 32:151-173, 2012

28. Samii M, Tatagiba M, Piquer J, Carvalho GA: Surgical treatment of epidermoid cysts of the cerebellopontine angle. $\mathbf{J}$ Neurosurg 84:14-19, 1996

29. Tatagiba M, Iaconetta G, Samii M: Epidermoid cyst of the cavernous sinus: clinical features, pathogenesis and treatment. Br J Neurosurg 14:571-575, 2000

30. Tomlinson BE, Walton JN: Granulomatous meningitis and diffuse parenchymatous degeneration of the nervous system due to an intracranial epidermoid cyst. J Neurol Neurosurg Psychiatry 30:341-348, 1967

31. Wang M, Li G, Jia D, Shen J: Clinical characteristics and surgical outcomes of patients with interdural epidermoid cyst of the cavernous sinus. J Clin Neurosci 20:53-56, 2013

32. Yanamadala V, Lin N, Walcott BP, Baird LC, Smith ER: Spontaneous regression of an epidermoid cyst of the cavernous sinus. J Clin Neurosci 21:1433-1435, 2014

33. Zhou LF, Mao Y, Chen L: Diagnosis and surgical treatment of cavernous sinus hemangiomas: an experience of 20 cases. Surg Neurol 60:31-37, 2003

34. Zhou LF, Mao Y, Zhang R: Surgical treatment of dumbbellshaped neurinomas: report of an experience with 57 cases in a single hospital. Surg Neurol 68:594-602, 2007

\section{Disclosures}

The authors report no conflict of interest concerning the materials or methods used in this study or the findings specified in this paper.

\section{Author Contributions}

Conception and design: Song, Zhu. Acquisition of data: Song, Zhou, Yang, Quan, S Li, P Li, Pan, Liu. Analysis and interpretation of data: Song, Zhou, Yang. Drafting the article: Song, Zhou, Yang. Critically revising the article: Song, Zhou, Zhu, Chen, Quan. Reviewed submitted version of manuscript: Song, Yang, Zhu, Chen, S Li, P Li, Pan, Liu, Mao. Approved the final version of the manuscript on behalf of all authors: Song. Administrative/ technical/material support: Song, Chen. Study supervision: Song, Zhu, Chen, Mao.

\section{Correspondence}

Jianping Song, Department of Neurosurgery, Huashan Hospital, Fudan University, No. 12 Wulumuqi Zhong Rd., Shanghai 200040, People's Republic of China. email: neurosurgerysong@ foxmail.com. 